\title{
LA PRESENCIA Y AUSENCIA DEL PRONOMBRE PERSONAL
}

Hu-Chuan Lu

Departamento de español, Universidad de Providencia

\section{INTRODUCCIÓN}

En los últimos años, el estudio del parámetro del sujeto nulo, en que la posición del sujeto puede estar ocupada o vacía, ha merecido la atención de los lingüistas de la sintaxis generativa. Por otra parte, las explicaciones del pronombre nulo propuestas por los variacionistas incluyen el estudio del español de Los Ángeles por Silva-Corvalán (1982), el del español portorriqueño por Hochberg (1986), el del español andaluz por Ranson (1991), el del portugués brasileño por Paredes Silva (1993) y el del español de San Juan (de Puerto Rico) y Madrid por Cameron (1993). En estos análisis, varios grupos de variables tanto linguiísticas como contextuales han sido estudiados; por ejemplo, la ambigüedad de la desinencia verbal, la referencia del sujeto, la ambiguiedad del discurso, etc. Sin embargo, no hay estudios parecidos del chino ni estudios comparativos en este campo.

El propósito del presente trabajo es examinar la significación estadística de las diferentes variables en la ocurrencia de la presencia o ausencia de sujeto en el español y el chino, analizando datos españoles y chinos extraídos del relato El libro de arena y de la obra teatral La señorita de Trevelez, y sus tra- 
ducciones respectivas, y explicando los fenómenos observados desde los puntos de vista teórico y funcional. Mis hipótesis son que 1) las variables independientes afectan a la presencia $u$ omisión de sujeto en una lengua o la otra, y 2) existe relación interactiva entre los dos idiomas con respecto a las variables independientes. A través de este estudio, procuraré contestar al siguiente planteamiento: si el español y el chino son dos lenguas que admiten el sujeto nulo, ¿se omite el sujeto en el mismo contexto en ambas lenguas?

\section{248 Datos CON VARBRUL}

Las teorías de gramática generativa ya nos han explicado con amplitud sobre el sujeto nulo en el español y el chino; no obstante, el descubrimiento de las funciones de sujeto en las estructuras superficiales parecidas es la motivación de hacer esta investigación. La descripción, el análisis y el cálculo del resultado estadístico de la presencia o ausencia del sujeto en los textos de las obras traducidas del español al chino serán los métodos objetivos para cumplir la meta.

\subsection{Datos}

Los 248 datos han sido extraídos del relato El libro de arena, de Jorge Luis Borges y de su respectiva traducción al chino. Este texto consta de cinco páginas y es del estilo de narración y diálogo.

\subsection{Método}

Se usa el programa de ordenador VARBRUL para examinar la significación estadística de la variable independiente, referente del sujeto.

Variable dependiente:

Sujeto

1. Presencia de sujeto.

2. Omisión de sujeto.

Variable independiente:

Referencia del sujeto

1. Sujeto correferencial con el sujeto en la oración anterior.

2. Cambio de referencia. 
Se usa el programa computacional VARBRUL (Checktok, Readtok, Makecell y Ivarb) para examinar el porcentaje y la significación estadística de la interacción del sujeto nulo y una variable independiente a través de cinco archivos creados: archivo de datos, archivo de especificación, archivo de condición, archivo de unidad y archivo de celdas.

Los datos españoles y los chinos son ejecutados en dos procesos separados en los cuales unas variables deben eliminarse en una lengua o la otra por razones estadísticas. Como consecuencia de todo esto, sólo 89 de 124 datos españoles y 117 de 124 datos chinos son aceptados en el cálculo final.

\subsection{Resultado}

El Cuadro 1, sorprendentemente, señala la gran diferencia de porcentaje en el cumplimiento de la omisión del sujeto en las dos lenguas en el mismo contexto. En el chino se prefiere la expresión del sujeto (73\%) al contrario que en el caso del español (10\%).

CUADRo 1. OMISIÓN Y PRESENCIA DEL SUJETO EN EL ESPAÑol Y EL CHINO

\begin{tabular}{|lcccc|}
\hline & español & \multicolumn{3}{c|}{ chino } \\
& $\mathbf{N}$ & $\%$ & $\mathbf{N}$ & $\%$ \\
Omisión de sujeto & $80 / 89$ & $90 \%$ & $32 / 117$ & $27 \%$ \\
Presencia de sujeto & $9 / 89$ & $10 \%$ & $85 / 117$ & $73 \%$ \\
\hline
\end{tabular}

El Cuadro 2 demuestra que en los datos estudiados del español, la variable independiente "referencia» prefiere la omisión del sujeto en el caso de que el referente del sujeto omitido es correferencial con el sujeto en la oración anterior (98\%), mientras que en el chino se prefiere la presencia del sujeto con el cambio de referentes $(89 \%)$.

Cuadro 2. Distribución Del sujeto en el español Y El Chino SEgún LAS VARIABLES INDEPENDIENTES «REFERENCIA» $Y$ «TIPO DE CLAUSULA»

\begin{tabular}{|lcccc|}
\hline & $\begin{array}{c}\text { español } \\
\text { Presencia }\end{array}$ & Omisión & Presencia & Omisión \\
Referencia correlacional & & & & \\
con el sujeto en la & $1 / 46(2 \%)$ & $\mathbf{4 5 / 4 6 ( 9 8 \% )}$ & $34 / 60(57 \%)$ & $26 / 60(43 \%)$ \\
oración anterior & $8 / 43(19 \%)$ & $35 / 43(81 \%)$ & $51 / 57(89 \%)$ & $6 / 57(11 \%)$ \\
cambio de referencia & & & & \\
\hline
\end{tabular}




\section{1000 Datos con Proc Catmod}

El resultado estadístico inesperado de la sección anterior estimula la investigación más profunda. En esta sección, se usan diferente programa computacional y diferentes datos para examinar la hipótesis propuesta en la sección 1.

\subsection{Datos}

En los trabajos traducidos, el teatro moderno tiene las formas de narración y diálogo que corresponden el objeto de este estudio. Los 500 datos españoles están extraídos de la primera a la quinta escena del primer acto de La señorita de Trevelez, cuyo autor es Carlos Arniches (1866-1943). Y los 500 datos chinos se sacan de las frases paralelas traducidas del español al chino por los estudiantes del Postgrado de la Universidad de Fu-Jen (1989).

\subsection{Métodos}

Considero las variables (de Grupo 2-Grupo 8) señaladas abajo como una lista posible de las variables independientes que pueden afectar la variable dependiente - la expresión u omisión del sujeto- en español y chino.

Variable dependiente:

Grupo 1: Sujeto.

1. Presencia de sujeto.

2. Omisión de sujeto.

Variables independientes:

Grupo 2: Referencia del sujeto.

1. Sujeto correferencial con el sujeto en la oración anterior.

2. Sujeto correferencial con el objeto indirecto en la oración anterior.

3. Sujeto correferencial con el objeto directo en la oración anterior.

4. Cambio de referencia.

Grupo 3: Ambigüedad morfologica de la forma verbal y del pronombre reflexivo.

1. Ambigüedad morfológica.

2. No ambigüedad morfológica. 
Grupo 4: Ambigüedad contextual.

1. Referente ambiguo en el discurso contextual.

2. Referente no ambiguo en el discurso contextual.

3. Referente identificado por la flexión verbal o el pronombre reflexivo.

Grupo 5: Contraste.

1. Sujeto contrastado.

2. Sujeto no contrastado.

Grupo 6: Tipo de sujeto.
1. Yo.
2. Tú.
3. Usted.
4. El/Ella.
5. Nosotros.
6. Vosotros.
7. Ustedes.
8. Ellos/Ellas.
9. Sintagma Nominal.

Grupo 7: Tipo de cláusula.

1. Cláusula independiente.

2. Cláusula coordinada.

3. Cláusula relativa.

4. Cláusula subordinada.

Grupo 8: Tipo de oración.

1. Oración declarativa.

2. Oración interrogativa.

3. Oración exclamativa.

4. Oración imperativa.

Los datos españoles y los chinos compilados son ejecutados en el programa de ordenador Proc Catmod de SAS 6.0 para examinar la significación estadística (valor de P) de la relación interactiva ${ }^{1}$ entre la variable dependiente -la presencia u omisión de sujeto-y las variables independientes - los ocho grupos de variables- en los dos idiomas y calcular el porcentaje de diferentes niveles de las variables independientes en la misma lengua. Un grupo de factor o un nivel de variable independiente no se aplica en una lengua o la otra por razones de propiedades de una u otra lengua; por ejemplo, la variable «ambi-

1 Es una relación cruzada, no paralela. 
güedad morfológica» no se considera en el caso del chino, mientras que sí se considera en el caso del español debido a la gran diferencia morfológica entre los dos idiomas. Además, observamos que en vez de los pronombres «Ud.» y «Uds.» en el español, se traducen «tú» y «vosotros» en el chino.

\subsection{Resultados}

La distribución de los datos según los diferentes grupos de variables independientes en el chino y el español se señala a continuación en el Cuadro 3.

CuAdro 3. DistRIBUCIÓN DE LOS DATOS SEGÚN LAS VARIABLES INDEPENDIENTES

\begin{tabular}{|llrr|}
\hline & & español & chino \\
Referencia & correferencial con sujeto en la oración anterior & 153 & 209 \\
& correferencial con objeto indirecto en la & 1 & 0 \\
& $\quad$ oración anterior & & \\
& cambio de referencia & 346 & 291 \\
Contexto & ambiguo en el discurso & 83 & 179 \\
& no ambiguo en el discurso & 138 & 321 \\
& referente identificado por inflexión & 279 & 0 \\
Contraste & sujeto contrastado & & \\
& sujeto no contrastado & 24 & 19 \\
& & 476 & 481 \\
Sujeto & yo & & \\
& tú & 157 & 155 \\
& usted & 58 & 134 \\
& élella & 65 & 0 \\
& nosotros & 88 & 86 \\
& vosotros & 35 & 32 \\
& ustedes & 17 & 23 \\
& ellos/ellas & 4 & 0 \\
& SN & 19 & 13 \\
& & 57 & 57 \\
Cláusula & independiente & & \\
& coordinante & 301 & 260 \\
& & 89 & 159 \\
\hline
\end{tabular}




\begin{tabular}{|rlrr|}
\hline & relativa & 24 & 5 \\
& subordinante & 86 & 76 \\
Oracion & & & \\
& declarativa & 361 & 381 \\
& interrogativa & 59 & 45 \\
& exclamativa & 41 & 44 \\
& imperativa & 39 & 30 \\
\hline
\end{tabular}

Aunque el español y el chino se consideran como lenguas que permiten sujeto nulo, el Cuadro 4, conforme a lo observado en la sección 2.3., señala la gran diferencia de porcentaje $(65,8 \%$ frente a $18,2 \%)$ en la omisión del sujeto en las dos lenguas. En el chino se prefiere la expresión del sujeto $(81,2 \%)$ al contrario que en el caso del español $(34,2 \%)$.

CUADRo 4. OMISIÓN Y PRESENCIA DEL SUJETO EN EL ESPAÑOL Y EL CHINO

\begin{tabular}{|lcccc|}
\hline & español & \multicolumn{3}{c|}{ chino } \\
& $\mathrm{N}$ & $\%$ & $\mathrm{~N}$ & $\%$ \\
Presencia del sujeto & 171 & 34,2 & 409 & $\mathbf{8 1 . 2}$ \\
Omision del sujeto & 329 & $\mathbf{6 5 , 8}$ & 91 & 18.2 \\
\hline
\end{tabular}

El Cuadro 5 demuestra que las variables independientes «referencia de sujeto», «ambigüedad contextual» $y$ «tipo de oración» son las que tienen la interacción con el lenguaje. La relación interactiva se puede atribuir a tres razones. Primero, la variable independiente afecta en una lengua pero no en la otra. Segundo, si controlamos el lenguaje, la variable dependiente varía según la variable independiente. Tercero, si controlamos la variable independiente, la variable dependiente varía según la lengua. Por ejemplo, en el caso de «referencia de sujeto», el referente del sujeto discutido es lo mismo que el sujeto en la oración anterior, es decir, el sujeto discutido aparecerá en el chino mientras el sujeto se omitirá en el español. Este fenómeno nos señala que estos dos idiomas se comportan contrariamente con respecto a la misma variable independiente. En cuanto a las variables independientes «ambigüedad morfologica», «contraste de sujeto», «tipo de sujeto» y «tipo de cláusula», no se señala la significación probabilística en la interacción de variables independientes y lenguajes. 
CuAdro 5. INTERACCIÓN DE VARIABLES INDEPENDIENTES Y LENGUAS

\begin{tabular}{|lc|}
\hline Variables Dependientes & $\mathbf{P}$ \\
Referencia de sujeto & 0,004 \\
Ambigüedad contextual & 0,037 \\
Tipo de oración & $\mathbf{0 , 0 0 0}$ \\
\hline
\end{tabular}

En el presente trabajo, la probabilidad significativa es equivalente a o menor de 0,05 .

El Cuadro 6 señala que el español prefiere la omisión del sujeto en los casos con el mismo referente que la oración anterior $(74,5 \%)$, mientras que en el chino se expresa el sujeto en los casos con distintos referentes $(91,1 \%)$.

En cuanto a la variable independiente «ambigüedad contextual», la ambigüedad se define en estos términos: sin la presencia del sujeto, si el sujeto no se puede recuperar por otras informaciones contextuales, el referente del sujeto se considera ambiguo; es decir, aunque el referente del sujeto no se puede recuperar ni de la inflexión de la forma verbal ni del pronombre, uno puede deducirlo del discurso contextual. En el chino, se permite la omisión del sujeto cuando el discurso contextual puede recuperar el referente del sujeto. El contexto ambiguo juega un papel importante en la presencia del sujeto en el español $(80,7 \%)$; eso se observa aún más claramente en el caso del chino $(99,4 \%)$.

Hablando de la variable independiente «contraste», en ambas lenguas los casos de los sujetos contrastados garantizan la presencia del sujeto $(100 \%)$.

Los Cuadros 7 y 8 señalan la distribución de la presencia y omision del sujeto con la variable «tipos de sujeto» que son ambos aplicables en el caso del español y del chino; sin embargo, no existe relación interactiva entre las dos lenguas. El Cuadro 7 señala que, en el español, todas las personas excepto «Ud.» $(90,8 \%)$ y «Uds.» $(75 \%)$ prefieren la omisión del sujeto. Además, la segunda y la tercera persona de plural «vosotros» y «ellos/ellas» (100\%) garantizan la omisión del sujeto. Por el contrario, en el caso del chino, como se ve en el Cuadro 8, la presencia del sujeto es favorecida por todas las personas.

En cuanto a la variable independiente «ambigüedad morfológica», se define como: sin la presencia del sujeto, si el referente del sujeto no se puede recuperar por las informaciones morfológicas, el referente del sujeto se considera como ambiguo. Para el término «ambigüedad morfologica», incluyo dos categorías: la desinencia de la forma verbal y el pronombre reflexivo. La flexión de los verbos de la primera y la tercera persona del singular en los casos del condicional, imperfecto, subjuntivo, condicional perfecto y pluscuamperfecto es ambigua; por ejemplo, el verbo «decía» puede tener el sujeto de la pri- 
CuAdro 6. Distribución DEL SUJETO NULO EN EL ESPAÑol Y EL CHINO SEGÚN LAS VARIABLES INDEPENDIENTES QUE TIENEN RELACIÓN INTERACTIVA CON LA LENGUA

\begin{tabular}{|lcccccc|}
\hline & $\begin{array}{c}\text { español } \\
\text { Presencia }\end{array}$ & $\begin{array}{c}\text { Omisión } \\
\text { Total }\end{array}$ & P & $\begin{array}{c}\text { chino } \\
\text { Presencia }\end{array}$ & Omisión & P \\
Referencia del sujeto & $171(34,2)$ & $329(65,8)$ & & $409(81,8)$ & $91(18,2)$ & \\
Sujeto de la oración anterior & $39(25,5)$ & $114(74,5)$ & & $144(68,9)$ & $65(31,1)$ & \\
C.I. de la oración anterior & $1(100)$ & $0(0)$ & & & & \\
Cambio de referente & $131(37,9)$ & $215(62,1)$ & & $265(91,1)$ & $26(8,9)$ & \\
& & & & & & \\
Ambigiedad contextual & & & 0,000 & & & 0,000 \\
Ambiguo en el discurso & $\mathbf{6 7 ( 8 0 , 7 )}$ & $16(19,3)$ & & $178(99,4)$ & $1(0,6)$ & \\
No ambiguo en el discurso & $49(35,5)$ & $89(64,5)$ & & $231(72)$ & $90(28)$ & \\
Identificado por la inflexión & $55(19,7)$ & $224(80,3)$ & & & & \\
Contraste & & & & & & $0,020^{*}$ \\
Contraste de focus & $24(100)$ & $0(0)$ & & $19(100)$ & $0(0)$ & \\
No contraste & $147(30,9)$ & $329(69,1)$ & & $390(81,1)$ & $91(18,9)$ & \\
\hline
\end{tabular}

* Significa que $\mathrm{P}$ es derivado y examinado por la prueba exacta de Fisher.

Cuadro 7. Distribución Del suJeto en el ESPañol SEgún LA VARIABLE INDEPENDIENTE «TIPO DE SUJETO»

\begin{tabular}{|llccc|}
\hline $\begin{array}{l}\text { Variables } \\
\text { Sujeto }\end{array}$ & Dependientes & Presencia & Omisión & P \\
& & & & 0,000 \\
& yo & $37(23,6)$ & $122(76,4)$ & \\
& tú & $6(10,3)$ & $52(89,7)$ & \\
& Ud. & $\mathbf{5 9}(90,8)$ & $6(9,2)$ & \\
& él/ella & $8(9,1)$ & $80(90,2)$ & \\
& nosotros & $2(5,7)$ & $33(94,3)$ & \\
& vosotros & $0(0)$ & $17(100)$ & \\
& Uds. & $\mathbf{3 ( 7 5 )}$ & $1(25)$ & \\
& ellos/ellas & $0(0)$ & $19(100)$ & \\
& SN & $57(100)$ & $0(0)$ & \\
\hline
\end{tabular}


Cuadro 8. Distribución del sujeto en el chino según

LA VARIABLE INDEPENDIENTE «TIPO DE SUJETO»

\begin{tabular}{|llccc|}
\hline Factor & Variable & Presencia & Omisión & Valor de P \\
Sujeto & & & & 0,000 \\
& yo & $\mathbf{1 4 2}(\mathbf{9 1 , 6 )}$ & $13(8,4)$ & \\
& tú & $\mathbf{1 0 0}(\mathbf{7 4 , 6 )}$ & $34(25,4)$ & \\
& el/ella & $\mathbf{5 5}(64)$ & $31(36,1)$ & \\
& nosotros & $\mathbf{2 7}(\mathbf{8 4 , 4 )}$ & $5(15,6)$ & \\
& vosotros & $\mathbf{1 9}(\mathbf{8 2 , 6 )}$ & $4(17,4)$ & \\
& ellos/ellas & $\mathbf{9 ( 6 9 , 2 )}$ & $4(30,8)$ & \\
& SN & $\mathbf{5 7 ( 1 0 0 )}$ & $0(0)$ & \\
\hline
\end{tabular}

mera persona singular o la tercera persona singular, mientras que el verbo «cumplio" no es un ejemplo de ambigüedad morfologica, porque la desinencia del verbo denota que el sujeto es de la tercera persona singular. En el presente trabajo, adoptamos la definición más restricta de «ambigüedad»; además, incluyo la inflexión y el pronombre de la tercera persona singular y plural «se» como una variable ambigua cuando no se puede distinguir si el sujeto es «ellos», «ellas» o un sintagma nominal. El Cuadro 9 señala la variable que sólo influye en la lengua española. Para nuestra sorpresa, en el caso del español, la mayor parte de los sujetos están omitidos a pesar de la ambigüedad morfológica $(91,6 \%)$, y la mayor parte de los sujetos están presentes aunque no son ambiguos morfológicamente $(71,9 \%)$.

CuAdro 9. CONTRIBUCIÓN DE LA VARIABLE INDEPENDIENTE EN LA PRESENCIA Y OMISION DEL SUJETO EN EL ESPANTOL

\begin{tabular}{|lcccc|}
\hline \multicolumn{2}{|l}{$\begin{array}{l}\text { Variable Independiente } \\
\text { Morfología }\end{array}$} & Presencia & Omision & P \\
& Ambiguo & $25(8,4)$ & $\mathbf{2 7 2 ( 9 1 , 6 )}$ & \\
& No ambiguo & $\mathbf{1 4 6 ( 7 1 , 9 )}$ & $57(28,1)$ & \\
\hline
\end{tabular}

Otras variables independientes como «tipo de cláusula» y «tipo de oración» no señalan la significación estadística $(>0,05)$. Eso significa que la variable independiente no afecta a la dependiente significativamente; por eso, rechazamos la hipótesis de que los niveles «cláusula independiente, coordinante, relativa, subordinante» y «oración declarativa, interrogativa, exclamativa e im- 
perativa» jueguen papeles determinantes en la presencia u omisión del sujeto en el español.

Continuamos discutiendo las variables que sólo se aplican en el chino, como se observa en el Cuadro 10. Hay que prestar atención a la variable independiente «tipo de cláusula», ya que todos los tipos de cláusula prefieren más la presencia del sujeto $(86 \%, 67,9 \%, 100 \%, 92,1 \%)$. En cuanto a los tipos de oración, todos los tipos de oración -excepto «imperativa»- prefieren la presencia del sujeto en chino $(84,5 \%, 88,9 \%, 84,1 \%, 33,3 \%)$.

Cuadro 10. Contribución de las variables INDEPEndientes «TIPO DE CLÁUSULA» Y «TIPO DE ORACIÓN» EN EL CHINO

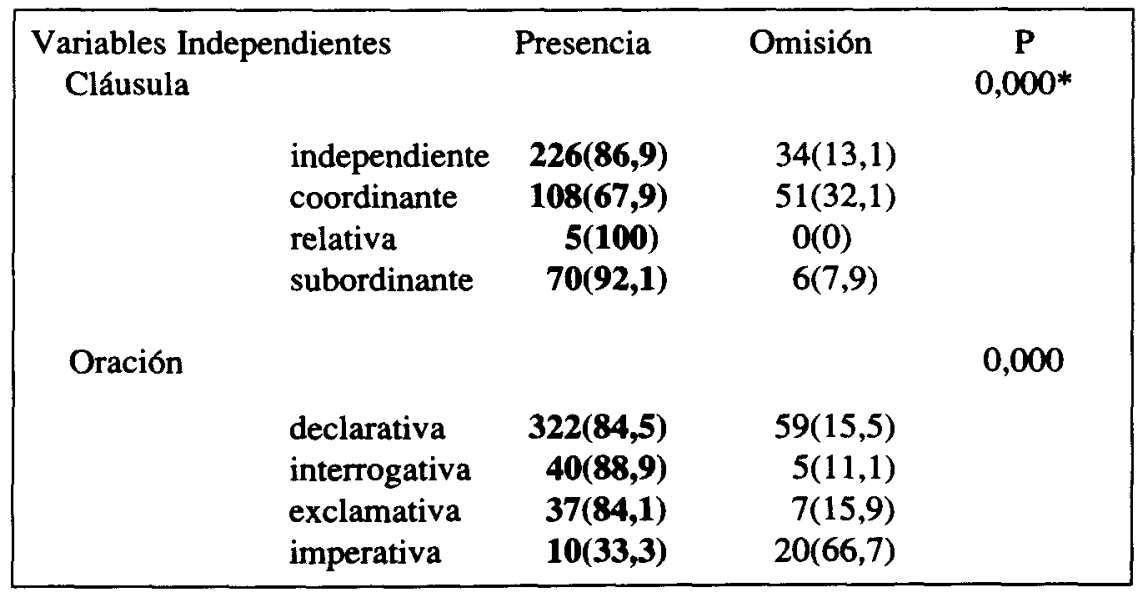

\section{Discusión}

Con el resultado de las secciones 2 y 3 , concluimos que, aunque el español y el chino son lenguas que permiten sujeto nulo, en realidad, el español prefiere la omisión del sujeto y el chino prefiere su presencia. Según la gramática generativa, la flexión es la causa principal de este contraste. Por ejemplo, la Hipotesis de Concordancia Rica de Taraldsen (1978) muestra que en español e italiano se permite sujeto nulo debido a su rica concordancia morfológica de sujeto-verbo, mientras el inglés no lo permite porque su sistema de concordancia es muy pobre. Si comparamos la flexión verbal del español y la del chino, encontramos la gran diferencia existente entre ambas lenguas, como se ve en (1) y (2). 
(1)

1 persona singular hablo

2 persona singular hablas

3 persona singular habla

1 persona plural hablamos

2 persona plural hablais

3 persona plural hablan

(2)

1 persona singular shuo 'digo'

2 persona singular shuo 'dices'

3 persona singular shuo 'dice'

1 persona plural shuo 'decimos'

2 persona plural shuo 'decís'

3 personal plural shuo 'dicen'

No obstante, al contrario de la Hipotesis de la Concordancia Rica, la rica concordancia morfológica de la forma verbal en el español no parece explicar la diferencia de la presencia y la ausencia de sujeto en el español: los sujetos prefieren aparecer a pesar de no haber ambigüedad morfológica y los sujetos prefieren omitirse a pesar de la existencia de ambigüedad de la flexión verbal. La riqueza de la flexión verbal (el único grupo de factor que no se aplica al chino) tampoco parece explicar el contraste del sujeto entre los dos idiomas. No es que neguemos la importancia de la morfología al poder recuperar los rasgos del pronombre personal omitido, sino que el resultado estadístico nos obliga a encontrar otros factores más importantes que la morfología.

De los resultados de la relación interactiva, adivinamos que las variables independientes «la referencia, ambigüedad contextual y tipo de oración» puedan tener su influencia. Sin embargo, como el valor de $P$ en la variable «tipo de oración» de español es menor de 0,05 , no la consideramos entre los factores. Primero, examinamos la variable de «referencia»: el español prefiere la omisión de sujeto cuando el referente del sujeto omitido es el mismo que el de la oración anterior, mientras que el chino prefiere la presencia en el caso de cambio de referente. Con esta observación, sugerimos que la «clarificación de comprensión» puede ser la causa que motiva la omisión de sujeto. Se puede explicar que el cambio de referente causa más confusión en la comprensión del sujeto en chino, y la facilidad de recuperación del sujeto de la oración anterior y la necesidad de evitar la confusión en la comprensión permiten la omisión del sujeto en español. Se puede concluir que la función lexica favorece la recuperación del sujeto. Esta idea coincide con las de Paredes Silva (1993), Ranson 
(1991), Silva-Corvalán (1982), Cameron (1993) y Hochberg (1986), en virtud de que tratan de la «compensación funcional» que representa el mantenimiento de información, que es semánticamente relevante y que tiende a expresar el pronombre funcional y estilísticamente.

Para probar la hipótesis de «clarificación de comprensión» en el discurso, se necesitan más evidencias. El resultado estadístico de la variable «ambigüedad contextual» puede explicar que la presencia del sujeto suele ocurrir cuando es posible la confusión de comprensión en español y en chino. El factor «contexto» verifica la importancia de «clarificación de comprensión» en el discurso tanto en el español como en el chino cuando se trata de determinar la presencia $o$ ausencia del sujeto.

Basándonos en Lu (1994), que opina que un pronombre nulo puede tener el mismo índice que el tópico cero o el sintagma nominal inmediato que lo ccomanda ${ }^{2}$; es decir, el tópico referido al discurso anterior o la frase anterior puede ser identificador para el sujeto nulo, como se ve en (3) a (6), añadimos que la «clarificación de comprensión» motiva la omisión de sujeto y la confusión de comprensión favorece la presencia de sujeto en español y chino. Las variables «la referencia» y «el contexto» aumentan la clarificación de comprensión y ofrecen evidencias y suplementan la teoría de «compensación funcional».

(3) (Hablando de Juan), $\emptyset$ es excelente. TÓPICO

(4) (Hablando de Juan $)$, mintian $\emptyset_{i}$ es muy excelente.

(5) (shuo dao Zhangsan ${ }_{i}$ ), mintian $\varnothing_{i}$ hui lai

hablar de Zhangsan, mañana va a venir

'(Hablando de Zhangsan ${ }_{i}$ ), (él) es excelente.'

(6) (shuo dao Zhangsan ${ }_{i}$ ), wo zhdao $\emptyset_{i}$ mingtian hui lai.

hablar de Zhangsan, yo sé mañana va a venir

'(Hablando de Zhangsan $\left.{ }_{\mathrm{i}}\right)$, sé que (él) es muy excelente.

En.cuanto a la variable «contraste», ambos idiomas garantizan la presencia del sujeto cuando el sujeto es contrastado. Eso se puede explicar por la propiedad del focus de que nunca puede expresar el énfasis sin aparecer el sujeto físicamente; es decir, el elemento focalizado no puede ser omitido. Como el focus que representa la información nueva, diferente que el tópico que represen-

${ }^{2}$ Chomsky (1981) propone que nodo A c-comanda nodo B cuando y sólo cuando

(i) A no domina B y B no domina A y

(ii) El primer nodo de ramas que domina A también domina $B$. 
ta la información conocida, debe ser visible para alcanzar el objeto que ha de ser contrastado. El factor «contraste» no entra en contradicción con la hipótesis de "clarificación de comprensión», porque el sujeto debe expresarse para demostrar su clarificación y, además, su importancia.

Hablando de la variable «los tipos de sujeto», el fenómeno observado en el caso del español justifica que la ambigüedad morfológica no juega un papel tan importante como creíamos; por ejemplo, a nuestro parecer, los pronombres de la tercera persona de singular «él», «ella» y «ellos», «ellas» causan más confusión y por eso deben aparecer con más frecuencia, aunque el resultado es el contrario. En cuanto a la segunda persona formal singular «Ud.» y «Uds.», ésta aparece más porque se debe enunciar cuando existe intención por parte del hablante de dirigirse a alguien. En el caso del chino, la primera persona singular, «yo», se expresa con más frecuencia, posiblemente con el fin de dar mayor énfasis para el contrastado «yo».

La variable «los tipos de la cláusula» sólo afecta al chino, pues el resultado estadístico señala que las cláusulas relativas y subordinantes prefieren la presencia del sujeto. Esto no contradice lo que se desarrolla en la gramática generativa, para la que la omisión del sujeto en la cláusula subordinada se recupera por la referencia de cláusula principal. Es necesario subrayar que el resultado observado aquí, junto con lo discutido para la variable «referencia», parece explicar que la barrera de jerarquía dificulta la comprensión de la referencia: si el sujeto omitido está en la cláusula subordinante o relativa, como se señala en (7), y no correferencia con el sujeto en la oración anterior, el sujeto omitido tiene, primero, que salir y montar SCOMP (Sintagma Complementario) que encaja el sujeto y, después, la oración principal para referirse a un sujeto en la oración anterior, mientras que es más fácil que el sujeto en la oración independiente y coordinante se correfieran con el sujeto en la oración anterior porque el nivel de la jerarquía es igual e idéntico, como se señala en (8) y (9), mientras que la jerarquía de la cláusula subordinante es más encajada. La «compensación funcional» también explica que las mismas propiedades de los elementos facilitan la «clarificación de comprensión».

Finalmente, en lo que respecta a la variable de «los tipos de oración» en chino, como el contexto es muy claro, se omite el sujeto en la oracion imperativa pero se expresa en las oraciones declarativa, interrogativa y exclamativa. En la oración imperativa, el sujeto referido siempre está claro en el discurso: si no es el oyente «tú» o «Ud.», debe ser un miembro del grupo de los oyentes «vosotros» o «Uds.». Por eso, no habrá confusión, y se permite omitir el sujeto con más frecuencia. Si comparamos la oración imperativa con otros tipos de oraciones, nos damos cuenta de que el referente de aquellas oraciones no es tan obvio como el de la oración imperativa. Como el discurso de las oraciones declarativas, interrogativas 
(7) Oración subordinante/relativa<smiles>N[SiH2]O[AlH2]</smiles>

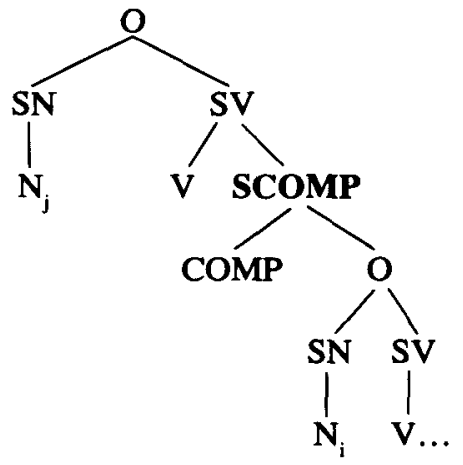

(8) Oración independiente<smiles>[AlH][AlH]1N[AsH]O1</smiles><smiles>N1[Al][AlH]O[SiH2]1</smiles><smiles>N1[Al][AlH]O[AsH]1</smiles>

(9) Oración coordinante<smiles>C[As]O[Si]N</smiles>

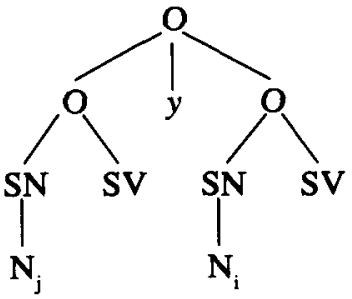

y exclamativas generalmente se refiere a una persona o describe algo relacionado con el hablante o el oyente, no está claro quién sea el referente del sujeto. Eso refuerza la propuesta de la «clarificación de comprension». Aunque los sujetos se expresan más en chino, en el caso de imperativo se omiten más frecuentemente.

\section{Conclusión}

Los resultados de los programas computacionales Varbrul y Proc Catmod de SAS 6.0 ofrecen evidencias de que la expresión de sujeto es más favorable 
en el chino, aunque esta lengua permite que la posición del sujeto esté vacía como en el español.

Los pesos probabilísticos señalan que existe relación interactiva entre las dos lenguas con respecto a las variables «referencia», "contexto» y «contraste». A pesar de la diferente preferencia en la expresión del sujeto en el español y el chino, las variables «ambigüedad contextual» $y$ «contraste» motivan el sujeto expresado en ambas lenguas. La propuesta de «clarificacion de comprensión» parece funcionar para explicar la presencia y ausencia de los sujetos en español y chino $y$, al mismo tiempo, refuerza la «compensación funcional» propuesta en los estudios anteriores.

Esperamos que lo discutido y observado en este trabajo nos ayude a entender las semejanzas y las diferencias existentes en dos lenguas llamadas «lenguas de sujeto nulo», desde los puntos de vista funcional y generativo.

\section{REFERENCIAS BIBIOGRÁFICAS}

CAMERON, RichaRd. 1993. «Ambiguous agreement, functional compensation, and nonspecific $t u ́$ in the Spanish of San Juan, Puerto Rico, and Madrid, Spain». Language Variation and Change, 5: 305-334.

- 1995. «Accessibility theory in a variable syntax». Conference on Functional Approaches to Grammar. UNM, Alburquerque.

Campos, H. 1992. «Silent objects \& subjects in Spanish». En Campos \& MartínezGil (ed.). Current Studies in Spanish Linguistics. Washington D. C.: Georgetown University Press.

Chomsky, Noam. 1981. Lecture on Government and Binding. Dordrecht: Foris Publications.

HOCHBERG, JUDITH G. 1986. «Functional compensation for /s/ deletion in Puerto Rican Spanish». Language, 62: 609-621.

JAEGGLI, O. \& SAIR, K. 1989. "The null subjet parameter and parametric theory». En Jaeggli, O. and Safir, K. (eds.). The Null Subject Parameter. Dordrecht: Kluwer Academic Publishers.

LAVANDERA, BEATRIZ. 1984. Variación y significado. Buenos Aires: Hachette.

Li, C. \& Thompson, S. 1981. Mandarin Chinese: a Functional Reference Grammar. Berkeley and Los Ángeles: University of California Press.

LÓPEZ MORALES, HUMBERTO. 1994. Métodos de investigación lingüística. Salamanca: Ediciones Colegio de España.

Lu, Hul-ChUAN. 1994. Preverbal NPs in Spanish and Chinese. UCLA Ph.D Dissertation.

Paredes Suva, Vera LuCla. 1993. «Subject omission and functional compensation: evidence from written Braxilian Portuguese». Language Variation and Change, 5: 35-49. 
Ranson, Diana L. 1991. «Person marking in the wake of /s/ deletion in Andalusian Spanish». Hispania, 76: 919-930.

Silva-Corvalán, Carmen. 1982. "Subjet expression and placement in MexicanAmerican Spanish». En Amastae, Jon, \& Elias-Olivares, L. (eds.). Spanish in the United States: Sociolinguistic Aspects. Vol. 3. Suppl. 2. 163-176.

- 1989. Sociolingüistica: teoría y análisis. Madrid: Alhambra.

- 1994. Sociolingüística: teoría y análisis. Madrid: Alhambra. Oxford: Clarendon.

TARAlSEN, T. 1978. On the NIC, Vacous Application, and the that-Trace Filter. Bloomington: Indiana University Linguistics Club. 